\title{
Phytoprotection
}

\section{Mode d'action des rhizobactéries favorisant la croissance des plantes et potentiel de leur utilisation comme agent de lutte biologique}

\section{C.J. Beauchamp}

Volume 74, numéro 1, 1993

URI : https://id.erudit.org/iderudit/706033ar

DOI : https://doi.org/10.7202/706033ar

Aller au sommaire du numéro

Éditeur(s)

Société de protection des plantes du Québec (SPPQ)l

ISSN

0031-9511 (imprimé)

1710-1603 (numérique)

Découvrir la revue

Citer cet article

Beauchamp, C. (1993). Mode d'action des rhizobactéries favorisant la croissance des plantes et potentiel de leur utilisation comme agent de lutte biologique. Phytoprotection, 74(1), 19-27. https://doi.org/10.7202/706033ar
Résumé de l'article

Les rhizobactéries qui favorisent la croissance des plantes, connues sous le terme RFCP, stimulent directement la croissance de celles-ci en augmentant le prélèvement des éléments nutritifs du sol, en induisant et produisant des régulateurs de croissance végétale et en activant les mécanismes de résistance induite chez les végétaux. Les RFCP stimulent indirectement la croissance des végétaux par leur effet antagoniste sur la microflore qui leur est néfaste, en transformant les métabolites toxiques et en stimulant la nodulation des légumineuses par les rhizobia. Les effets antagonistes des RFCP impliquent la production d'antibiotiques et la compétition nutritionelle avec les pathogènes végétaux. L'établissement de l'association RFCP-plante est primordiale pour l'expression des effets bénéfiques aux plantes. L'utilisation des RFCP marquées avec des gènes de bioluminescence permet de visualiser le processus de colonisation racinaire. Suite à l'apparition des exsudats de la semence, l'inoculum bactérien se multiplie, puis les bactéries sont transportées passivement par la racine en développement, hors de la zone d'influence de la semence. Par la suite, les RFCP continuent de se multiplier grâce aux exsudats racinaires et persistent sur les racines. Plusieurs compagnies développent actuellement des inoculants contenant des RFCP, surtout afin de réduire l'utilisation des pesticides de synthèse en agriculture.
Ce document est protégé par la loi sur le droit d'auteur. L'utilisation des services d'Érudit (y compris la reproduction) est assujettie à sa politique d'utilisation que vous pouvez consulter en ligne.

https://apropos.erudit.org/fr/usagers/politique-dutilisation/ 


\title{
Mode d'action des rhizobactéries favorisant la croissance des plantes et potentiel de leur utilisation comme agent de lutte biologique
}

\author{
Chantal J. Beauchamp ${ }^{1}$
}

Reçu 1992-11-18; accepté 1993-05-11

\begin{abstract}
Les rhizobactéries qui favorisent la croissance des plantes, connues sous le terme RFCP, stimulent directement la croissance de celles-ci en augmentant le prélèvement des éléments nutritifs du sol, en induisant et produisant des régulateurs de croissance végétale et en activant les mécanismes de résistance induite chez les végétaux. Les RFCP stimulent indirectement la croissance des végétaux par leur effet antagoniste sur la microflore qui leur est néfaste, en transformant les métabolites toxiques et en stimulant la nodulation des légumineuses par les rhizobia. Les effets antagonistes des RFCP impliquent la production d'antibiotiques et la compétition nutritionelle avec les pathogènes végétaux. L'établissement de l'association RFCP-plante est primordiale pour l'expression des effets bénéfiques aux plantes. L'utilisation des RFCP marquées avec des gènes de bioluminescence permet de visualiser le processus de colonisation racinaire. Suite à l'apparition des exsudats de la semence, l'inoculum bactérien se multiplie, puis les bactéries sont transportées passivement par la racine en développement, hors de la zone d'influence de la semence. Par la suite, les RFCP continuent de se multiplier grâce aux exsudats racinaires et persistent sur les racines. Plusieurs compagnies développent actuellement des inoculants contenant des RFCP, surtout afin de réduire l'utilisation des pesticides de synthèse en agriculture.
\end{abstract}

Beauchamp, C.J. 1993. Mode of action of plant growth-promoting rhizobacteria and their potential use as biological control agent. PHYTOPROTECTION 74: 19-27.

Plant growth-promoting rhizobacteria, known as PGPR, stimulate directly plant growth by increasing plant nutrients uptake, by inducing and producing plant growth regulators and by activating the induced resistance mechanisms in plants. PGPR stimulate indirectly plant growth by their antagonistic effect on detrimental microflora, by transforming toxic metabolites and by stimulating the nodulation of leguminous plants by rhizobia. The PGPR antagonistic effects is due to the production of antibiotics and to the competition for nutrients with plant pathogens. The establishment of the PGPR-plant association is essential for the expression of beneficial effects to plants. Using PGPR marked with bioluminescence genes allows to observe the root colonization process. Following the appearance of seed exudates, the bacterial inoculum reproduces and the bacteria are passively carried away from the seed zone with the developing root. Subsequently, PGPR continue to reproduce following stimuli from the root exudates and remain on the roots. Many companies are developing inoculants containing PGPR, in order to reduce the use of synthesized pesticides in agriculture.

1. Département des sols, Faculté des sciences de l'agriculture et de l'alimentation, Université Laval, Québec (Québec), Canada G1K 7P4 


\section{INTRODUCTION}

La rhizosphère est la zone de sol qui est sous l'influence des exsudats racinaires. Dans cette zone se trouve un groupe particulier de bactéries, les rhizobactéries. Ces dernières sont capables de se multiplier et de rivaliser avec les autres microorganismes pour occuper cette zone riche en éléments nutritifs. L'association, le rôle et les effets que les rhizobactéries exercent sur la plante sont fonction du succès de leur établissement dans la rhizosphère; elles peuvent avoir un effet positif, négatif ou neutre sur la croissance des plantes. Près de $5 \%$ des rhizobactéries favorisent la croissance des plantes et les protègent contre les agents pathogènes tels les bactéries, les champignons (Suslow 1982; Weller 1988) et les nématodes (Kloepper et al. 1992). L'inoculation des semences avec ces rhizobactéries se traduit généralement par des accroissements de rendement d'environ 10 à 30\% (Suslow 1982). Ces rhizobactéries appartiennent à différents groupes taxonomiques de bactéries. Elles ont été regroupées sous le nom de Rhizobactéries qui Favorisent la Croissance des Plantes (RFCP). Les RFCP les plus fréquemment identifiées sont des pseudomonades fluorescentes, mais comptent aussi des Bacillus, des Azospirillum, des Azotobacter, des Klebsiella, des Enterobacter, des Rhizobium et des Serratia spp. (Bakker et al. 1991; Kloepper 1992).

\section{LES MODES D'ACTION DIRECTS}

Certaines RFCP stimulent la croissance des plantes en l'absence de pathogènes. Ces effets directs regroupent les accroissements de la masse aérienne et racinaire, les élongations racinaires, et les levées accélérées des plantules. Ces augmentations s'expliquent généralement par de meilleurs prélèvement et assimilation des éléments nutritifs par la plante, la production de phytohormones et le développement de résistance induite chez les plantes.

Une meilleure nutrition azotée est assurée par la symbiose Rhizobium-légumineuses, et par les associations Azospi- rillum-céréales, et Bacillus-céréales (Gaskins et al. 1985). Diverses bactéries ont la capacité de solubiliser le phosphore organique par l'action de phosphatase, ou le phosphore inorganique par la libération d'acides organiques (Kloepper et al. 1989). Les semences de canola (Brassica campestris L.) inoculées avec la RFCP, GR12-2, ont un contenu en phosphore supérieur aux témoins non-inoculés (Lifshitz et al. 1987).

Plusieurs microorganismes ont aussi la capacité de produire des auxines, des gibbérellines et des substances de type kinétines in vitro (Brown 1974; Frankenberger et Arshad 1991). Selon ces auteurs, les régulateurs de croissance qui sont produits dans la rhizosphère peuvent être prélevés directement par les racines et stimuler la croissance de la plante. Les quantités produites varient avec le microorganisme impliqué et la présence des précurseurs métaboliques des régulateurs de croissance. La L-tryptophane est le précurseur des auxines chez les microorganismes, dont des Azotobacter, des Azospirillum, des Rhizobium, des Pseudomonas et des Bacillus spp. En présence d'adénine et d'alcool isopentyle, certains de ces microorganismes produisent des kinétines. Finalement, plusieurs bactéries du sol, dont des Pseudomonas spp., vont produire de l'éthylène en présence de la $L$-méthionine ou la $L$-éthionine.

La résistance induite envers les pathogènes des plantes a été démontrée chez plus de 25 espèces végétales, et s'exprime par la diminution du nombre et de la dimension de lésions d'attaque par ces pathogènes (Kùc 1987). Certaines RFCP ont la capacité d'induire la résistance des plantes envers certains pathogènes (van Peer et al. 1991; Wei et al. 1991).

\section{LES MODES D'ACTION INDIRECTS}

Certaines RFCP produisent des effets bénéfiques sur la croissance des plantes en présence d'un pathogène ou d'un rhizobia. Ces modes d'action indirects sont généralement attribuables à la compétition, à la production d'antibiotiques et à la détoxification du milieu; chez les légumineuses, les RFCP mènent à l'ac- 
croissement du nombre de nodosités produites par les rhizobia (Grimes et Mount 1987; Polonenko et al. 1987). Par ailleurs, la levée plus hâtive des plantules et la croissance plus rapide des racines liées aux RFCP permettent aux plantes d'échapper plus rapidement aux pathogènes.

Les RFCP produisent des métabolites secondaires qui sont toxiques aux pathogènes du sol, dont les antibiotiques (Howell et Stipanovic 1979, 1980; Howie et Suslow 1991; Thomashow et Weller 1990), le cyanide (Schippers et al. 1991), les sidérophores (Demange et al. 1987; Leong 1986; Loper et Buyer 1991), et diverses substances qui ont une gamme d'activités inhibitrices de la croissance des pathogènes (Howell et al. 1988; Inbar et Chet 1991; Keel et al. 1992; Sneh 1981). L'activité de ces métabolites secondaires a été démontrée à l'aide de bactéries mutantes négatives pour la production d'un principe actif (Flaishman et al. 1990).

D'autres RFCP agissent comme hyperparasites des champignons pathogènes et produisent des exoenzymes dégradant la chitine, la cellulose ou les ß-glucanes lysant leur paroi. L'activité chitinolytique de certaines RFCP explique leur activité inhibitrice de certains champignons pathogènes (Inbar et Chet 1991). L'Arthrobactersp., une bactérie chitinolytique, lyse le mycélium du Fusarium oxysporum Schlecht. f. sp. dianthii Prill \& Del. (Sneh 1981), du Rizoctonia solani Kühn, du Sclerotium rolfsii Sacc. et du Fusarium oxysporum Schlecht. f.sp. vasinfectum Snyder \& Hansen (Inbar et Chet 1991).

Les phytotoxines sont des composés produits par des microorganismes saprophytes et parasitiques du sol et inhibent à de faibles concentrations la croissance et le développement des plantes. Leurs structures chimiques sont très variées. Dans le sol, ces toxines s'accumulent en quantité plus appréciable lors de monocultures. Certaines pseudomonades fluorescentes éliminent ces phytotoxines.

Une RFCP peut exprimer à la fois un mode d'action direct et indirect. Par exemple, la RFCP Pseudomonas putida (Trevisan) Migula souche 61.9A, a été sélectionnée pour favoriser la croissance du concombre (Cucumis sativus L.) en l'ab-

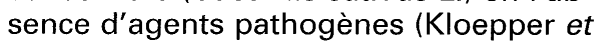
al. 1991). Par contre, lorsque ces plantes sont inoculées avec cette souche et mises en présence de Pythium, elles sont protégées des attaques de ce microorganisme.

\section{L'UTILISATION DES RFCP EN AGRICULTURE}

Actuellement, diverses formulations commerciales de RFCP sont en vente. Des formulations bactériologiques de Rhizobium spp. sont disponibles dans plusieurs pays afin de favoriser la nodulation des légumineuses et de diminuer la fertilisation azotée des cultures. La formulation Zea-nit Plus est en vente en Italie. II s'agit $\mathrm{d}^{\prime}$ un inoculum à base d'Azospirillum sp. développé pour inoculer le maïs (Zea mays L.) (J. W. Kloepper, communication personnelle). De même, la formulation Quantum 4000, à base de Bacillus subtilis (Ehremberf) Cohn, souche A13 (Broadbent et al. 1977), est commercialisée aux États-Unis pour lutter contre le Rhizoctonia solani Kühn chez l'arachide (Arachis hypogaea L.), le haricot (Phaseolus vulgaris L.) et le coton (Gossypium hirsutum L.). En Chine, des RFCP qui accroissent les rendements sont utilisées dans plusieurs cultures, et ce, depuis 1985 (J. W. Kloepper, communication personnelle). En 1987, 3,35 millions ha et plus de 48 cultures avaient été traités avec ces bactéries bénéfiques. La valeur des accroissements en rendement obtenus équivaut à plus de 60 millions de dollars canadiens.

Le problème le plus important qui rend le succès de l'implantation des RFCP incertain en agriculture provient de leur inconstance à promouvoir la croissance des plantes. Cette variabilité peut s'expliquer par les facteurs biotiques et abiotiques affectant la survie et les effets des RFCP dans la rhizosphère. II est donc important de comprendre les premières étapes de l'établissement de I'association RFCP-plante afin d'optimiser les succès de la colonisation racinaire et l'expression du principe actif. 


\section{LA COLONISATION RACINAIRE ET LE MARQUAGE GÉNÉTIQUE DES BACTÉRIES DE LA RHIZOSPHËRE}

En théorie, suite à l'inoculation de la semence, les RFCP se multiplient en réponse aux exsudats émis par la semence, s'établissent sur la racine en émergence, puis colonisent le système racinaire (Kloepper et al. 1989). Les RFCP persistent généralement jusqu'à la misaison de croissance de la plante-hôte en maintenant des populations de $10^{3}$ à $10^{6}$ unités par gramme de racine, chaque unité ayant la possibilité de former une colonie (Kloepper et al. 1991). Certaines RFCP auraient même la capacité de coloniser les portions internes des racines (van Peer et al. 1990). Ces bactéries sont dites RFCP-endophytes.

II existe une gamme de techniques utilisables pour dénombrer les microorganismes colonisateurs de la rhizosphère (Kloepper et Beauchamp 1992), mais le dénombrement sur gélose sélective demeure la technique la plus fréquemment utilisée. L'emploi de bactéries résistant spontanément à un antibiotique facilite leur quantification sur gélose enrichie de cet antibiotique. Cependant, ce type de marquage peut être perdu suite aux passages des bactéries dans un sol non-stérile (Lindström et al. 1990), et devenir insatisfaisant lorsque plusieurs souches sont à l'étude. La modification génétique de bactéries par l'insertion de gènes marqueurs est en développement pour palier cette lacune. Diverses études (Drahos et al. 1986; Höfte et al. 1990; Kluepfel et al. 1990; Seong et al. 1991) ont démontré l'utilité des gènes lacZ et lacZY pour identifier et dénombrer des Pseudomonas spp. ainsi marqués génétiquement, dans la rhizosphère de plusieurs espèces de plantes. Le gène lacZ est responsable de la synthèse de l'enzyme ßgalactosidase, et les colonies des bactéries transformées prennent une couleur bleue lorsqu'elle croissent sur une gélose contenant le réactif $X-G a l$ (5-chloro-4bromo-3-indolyl-ß-D-galactopyranoside). Ce marquage génétique offre la possibilité de discerner les bactéries véhiculant les gènes lac $Z$ des autres bactéries du sol.

\section{LA BIOLUMINESCENCE COMME MARQUEUR GÉNÉTIQUE}

Les gènes de la bioluminescence (/ux) offrent aussi un marquage génétique unique puisqu'ils confèrent à la bactérie marquée la propriété de produire des rayons lumineux. Ces gènes ont été clonés chez Vibrio fischeri (Beijerinck) Lehmann et Newmann, $V$. harveyi (Johnson et Shunk) Bang et Woolkalis, Photobacterium sp. et Xenorhabdus luminescens Thomas et Poinar (Belas et al. 1982; Delong et al. 1987; Engebrecht et al. 1983; Frackman et al. 1990). La capacité d'émettre de la bioluminescence chez les bactéries se trouve surtout chez des bactéries marines. Un Tn5-LuXAB a été construit qui véhicule les gènes de la luciférase de V. harveyi (Boivin et al. 1988). La rhizobactérie modifiée génétiquement avec les gènes luxAB (RMG-Lux) synthétise la luciférase, ce qui lui confère la capacité d'émettre de la bioluminescence en présence d'un acide gras à longue chaine (ex. décanal); il y a alors oxydation du $\mathrm{FMNH}_{2}$ et des photons sont émis (Meighen 1991). Les RMG-Lux présentent un nouveau phénotype qui les différencie des autres bactéries de la rhizosphère et du sol. Actuellement, aucune bactérie luminescente n'a été décelée sur les géloses utilisées pour isoler les rhizobactéries ou des Xanthomonas campestris (Pammel) Dowson (J.J. Shaw, communication personnelle). Le phénotype bactérien est donc unique pour les bactéries de la rhizosphère.

Les colonies de bactéries biolurninescentes peuvent être observées de diverses façons: visuellement dans une chambre noire, à l'aide de pellicule photographique (Beauchamp et al. 1991, 1993a, 1993b; Grant et al. 1991; Legocki et al. 1986; O'Kane et al. 1988) et à rayons X (de Wegger et al. 1991; Shaw et al. 1987) ou à l'aide d'une caméra spécialisée pour détecter des quantités faibles de photons (caméra CCD) (Beauchamp et al. 1993b; Mahaffee et al. 1991; Shaw et al. 1992). Des comptages photoniques peuvent 
aussi être obtenus à l'aide d'un compteur à scintillation (Boivin et al. 1988; Fravel et al. 1990; Mahaffee et al. 1991), d'un luminomètre (Shaw et al. 1987, 1992) et du programme statistique couplé à la caméra CCD (Shaw et al. 1992). Il est donc possible de suivre les bactéries bioluminescentes dans la rhizosphère de façon spécifique, soit quantitativement, soit qualitivement en visualisant les sites de colonisation du système racinaire.

Des études de colonisation racinaire en conditions stériles révèlent que différentes souches RMG-Lux ont la capacité de coloniser complètement tout le système racinaire (Figure 1). De plus, certaines parties aériennes, dont les tiges, sont aussi colonisées. Ce genre d'étude cependant ne permet pas de comprendre les phénomènes de colonisation racinaire en conditions non stériles. Pour ce faire, un modèle utilisant des rhizoboîtes

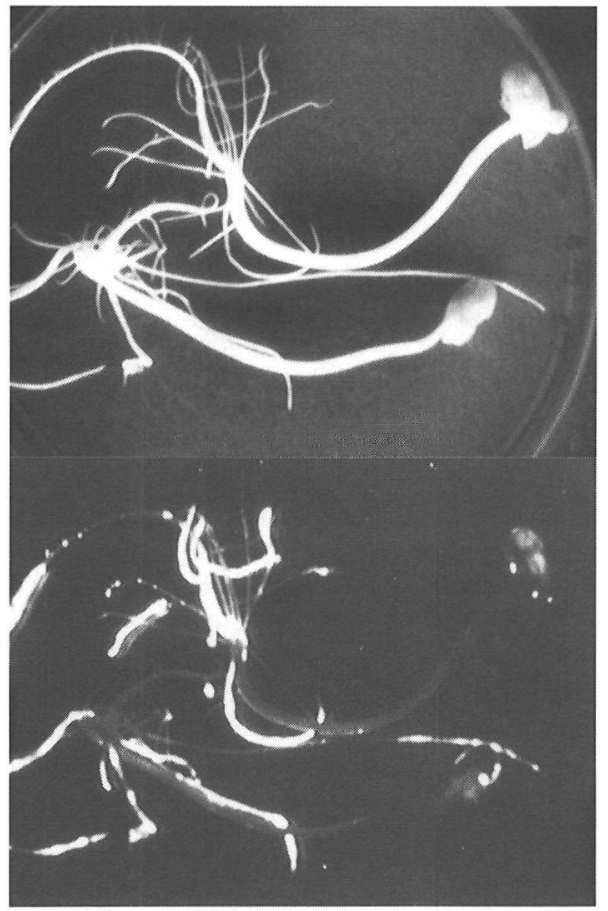

Figure 1. Distribution de la bactérie bioluminescente $86.139 \mathrm{~L}$ sur des plantules de soja cultivées dans des conditions stériles 8 j après I'inoculation de la semence. Vue illuminée par la lumière incidente (haut) et par la bioluminescence produite par les bactéries (bas). $(0,75 \mathrm{X})$ en plexiglas translucide a été développé pour étudier la colonisation racinaire dans des conditions similaires à celles retrouvées au champ (Beauchamp et al. 1991, 1993b). Ces rhizoboîtes contiennent du sol non stérile ajusté à la capacité au champ. La semence inoculée avec des RMG-Lux est placée dans ces rhizoboîtes et les phénomènes de colonisation de la spermosphère et de la rhizosphère peuvent être visualisés. En couplant les techniques de la rhizoboîte et de la bioluminescence, il est possible de suivre ainsi la dynamique de la colonisation racinaire. Les RMG-Lux peuvent être localisées grâce à une empreinte du système radiculaire sur une gélose sélective (C. J. Beauchamp, données non publiées), en disséquant stérilement le système racinaire et en plaçant les parties de racines dans une gélose sélective à environ 46 à $50^{\circ} \mathrm{C}$ (Beauchamp et al. 1991, 1993a), ou par photographie avec la caméra $C C D$ (Beauchamp et al. 1993b).

La colonisation racinaire du coton, du concombre, du maïs et du soja (Glycine max Merr.) a été démontrée par quatre souches différentes de RMG-Lux (Beauchamp et al. 1993b). Pour toutes les souches et les plantes à l'étude, la portion supérieure du système racinaire a été plus fortement colonisée que l'apex racinaire et, en plus grande quantité, la racine principale que les racines secondaires.

II a aussi été observé que la quantité de photons émise varie quand une souche est en compétition avec des champignons du sol (C. J. Beauchamp, données non publiées). La bioluminescence semble donc permettre d'évaluer l'état physiologique de la bactérie. Des études complémentaires devraient être entreprises pour mieux comprendre les variations dans ces phénomènes biologiques.

La bioluminescence permet de suivre la colonisation racinaire dans le temps. À l'aide des rhizoboîtes, il a été possible de visualiser la colonisation racinaire du soja suite à l'inoculation de la semence avec la RMG-Lux, souche GR7.4L (C. J. Beauchamp, données non publiées). Après la germination, les rhizobactéries ont migré des téguments de la graine vers l'extrémité de la radicule. Sept jours 
après l'inoculation de la semence, la quantité de RMG-Lux avait diminué sur les téguments. Cette baisse s'explique par la diminution des exsudats de la spermosphère et par le déplacement des téguments vers la surface du sol en compagnie de l'épicotyle. Les rhizobactéries bioluminescentes sont affectées négativement par les conditions sèches présentes dans la couche superficielle du sol et de l'air. Les rhizobactéries persistent sur les racines, cependant les racines étant plus longues, il $\mathrm{y}$ a un phénomène de dilution qui s'opère et la quantité de photons émise diminue avec le temps. II est possible d'observer dans la même rhizoboîte un système racinaire d'une plantule colonisée par les rhizobactéries bioluminescentes alors que l'autre plantule ne l'est pas. Dans ce cas particulier, il est difficile d'expliquer la raison de cette absence de colonisation racinaire puisque les conditions expérimentales sont similaires du semis à la prise de la photo. Des études détaillées permettraient d'identifier les facteurs affectant la colonisation racinaire. Le modèle de la rhizoboîte est limité dans le temps et il ne permet plus de suivre la colonisation lorsque les racines ont atteint l'extrémité inférieure de la rhizoboîte.

Pour étudier la colonisation racinaire en fonction du temps et des conditions environnementales prévalant au champ, la RMG-Lux, souche 61.9A.3L ( $P$. putida), a été relâchée dans l'environnement (Beauchamp et al. 1992). L'expérience s'est déroulée à Shorter, Alabama, U.S.A. suite à l'obtention d'un permis de courtoisie du USDA-APHIS. La semence de soja a été inoculée au laboratoire soit avec la souche $61.9 \mathrm{~A}$ résistante à la rifampicine, soit avec la souche bioluminescente 61.9A.3L, ou avec un mélange de ces deux souches dans un rapport 1:1. Un témoin sans bactérie a aussi été inclus. Les populations bactériennes ont été dénombrées pendant $70 \mathrm{j}$. Les rangs de soja étaient espacés de $45 \mathrm{~cm}$ et dans ces conditions, les bactéries bioluminescentes $n^{\prime}$ ont pas migré d'une parcelle à une autre. Les populations bactériennes et fongiques totales n'ont pas été affectées par l'inoculation de la semence. La population des rhizobactéries bioluminescentes a diminué dans le temps, et cette diminution a été beaucoup plus rapide lorsqu'elles étaient en mélange avec la souche 61.9A. Au champ, la souche 61.9A.3L a une moins bonne compétitivité que la souche 61.9A. La bioluminescence est une technique qui permet d'étudier les interactions rhizobactériesplantes dans des conditions environnementales normales. Aucune bactérie bioluminescente autre que celle introduite n'a été observée.

\section{L'AVENIR DES RFCP}

Les pressions sociales en vue de diminuer l'emploi des pesticides en agriculture accentuent la demande pour des produits biologiques aptes à contrer les pathogènes des plantes. II devient donc important de développer différentes méthodes de lutte biologique par I'utilisation d'organismes naturels ou modifiés génétiquement, ou le produit de leurs gènes, et ce, afin de réduire les effets d'un organisme néfaste et de favoriser les organismes utiles tels les cultures, les arbres, les animaux et les insectes et microorganismes bénéfiques.

L'utilisation des RFCP en agriculture peut permettre de stimuler la croissance des plantes et de contrer leurs pathogènes. L'emploi des RFCP modifiés génétiquement ou non peut permettre de diminuer I'utilisation des pesticides, ce qui devrait encourager les efforts de recherche dans ce domaine. Des combinaisons de RFCP ayant des modes d'action différents et colonisant différents sites racinaires doivent être étudiées. La libération de microorganismes modifiés dans l'environnement doit aussi s'intensifier et les procédures canadiennes et québécoises entourant l'obtention de permis doivent être simplifiées.

\section{REMERCIEMENTS}

L'auteure tient à remercier le Conseil de recherches en sciences naturelles et en génie (CRSNG) du Canada pour l'obtention des bourses d'études supérieures et post-doctorales, et les Départements de phytopathologie et de botanique et $\mathrm{mi}$ crobiologie de I'Université d'Auburn, Alabama, U.S.A., pour leur contribution financière à ces projets de recherches. 


\section{RÉFÉRENCES}

Bakker, P.A.H.M., R. van Peer et B. Schippers. 1991. Suppression of soil-borne plant pathogens by fluorescent pseudomonads: mechanisms and prospects. Pages 217230 in A. R. B. Beemster, G. J. Bollen, M. Gerlagh, M. A. Ruissen, B. Schippers et A. Tempel (réds.), Biotic interactions and soilborne diseases. Elsevier, New York.

Beauchamp, C.J., J.W. Kloepper et P.A. Lemke. 1991. Root colonization of various host plants by Tn 5 bioluminescent pseudomonads. Pages 243-247 in C. Keel, B. Koller et G. Défago (réds.), Plant growth-promoting rhizobacteria-progress and prospects. IOBC/WPRS Bull. XIV/8.

Beauchamp, C.J., J.W. Kloepper, J. J. Shaw et P.A. Lemke. 1992. Bioluminescence as a marking system to monitor the fate of Pseudomonas putida, strain 61.9A.3L, released in the soybean rhizosphere. Phytopathology 82: 1179 (résumé).

Beauchamp, C.J., J.W. Kloepper et H. Antoun. 1993a. Detection of genetically-engineered bioluminescent pseudomonads in potato rhizosphere at different temperatures. Microb. Releases 1: 200-207.

Beauchamp, C.J., J.W. Kloepper et P.A. Lemke. 1993b. Luminometric analysis of plant root colonization by bioluminescent pseudomonads. Can. J. Microbiol. 39: 434-441.

Belas, R., A. Mileham, D. Cohn, M. Hilmen, M. Simon et M. Silverman. 1982. Bacterial bioluminescence: isolation and expression of the luciferase genes from Vibrio harveyi. Science (Wash. DC) 218: 791-793.

Boivin, R., F.-P. Chalifour et P. Dion. 1988. Construction of a Tn 5 derivative encoding bioluminescence and its introduction in Pseudomonas, Agrobacterium, and Rhizobium. Mol. \& Gen. Genet. 213: 50-55.

Broadbent, P., K.F. Bakker, F. Franks et J. Holland. 1977. Effect of Bacillus spp. on increased growth of seedlings in steamed and nontreated soil. Phytopathology 67: 1027-1034.

Brown, M.E. 1974. Seed and root bacterization. Annu. Rev. Phytopathol. 12: 181-197.

Delong, E.F., D. Steinhauer, A. Israel et K.H. Nealson. 1987. Isolation of the lux genes from Photobacterium leiognathi and expression in Escherichia coli. Gene (Amst.) 54: 203-210.

Demange, P., S. Wendenbaum, A. Bateman, A. Dell et M.A. Abdallah. 1987. Bacterial siderophores: Structure and physicochemical properties of pyoverdins and related compounds. Pages 167-187 in G. Winkleman, D. van der Helm et J.B. Neilands (réds.), Iron transport in microbes, plants and animals. VCH Chemie, Weinheim, FRG. de Weger, L.A., P. Dunbar, W. Mahaffee, B.J.J. Lugtenberg et G.S. Sayler. 1991. Use of bioluminescence markers to detect Pseudomonas spp. in the rhizosphere. Appl. Environ. Microbiol. 57: 3641-3644.

Drahos, D.J., B.C. Hemming et S. McPherson. 1986. Tracking recombinant organisms in the environment: ß-galactosidase as a selectable non-antibiotic marker for fluorescent pseudomonads. Bio/Technol. 4: 439-444.

Engebrecht, J., K.H. Nealson et M. Silverman. 1983. Bacterial bioluminescence: isolation and genetic analysis of functions from Vibrio fischeri. Cell 32: 773-781.

Flaishman, M., Z. Eyal, C. Voisard et D. Hass. 1990. Suppression of Septoria tritici by phenazine- or siderophores-deficient mutants of Pseudomonas. Curr. Microbiol. 20: 121-124.

Frackman, S., M. Anhalt et K. H. Nealson. 1990. Cloning, organization, and expression of the bioluminescence genes of Xenorhabdus luminescens. J. Bacteriol. 172: 5767-5773.

Frankenberger, W.T., Jr. et M. Arshad. 1991. Microbial production of plant growth regulating substances in soil. Pages 162-171 in C. Keel, B. Koller et G. Défago (réds.), Plant growth-promoting rhizobacteriaprogress and prospects. IOBCNPRS Bull. $\mathrm{XIV} / 8$.

Fravel, D. R., R. D. Lumsden et D. P. Roberts. 1990. In situ visualization of the biocontrol rhizobacterium Enterobacter cloacae with bioluminescence. Plant Soil 125: 233-238.

Gaskins, M.H., S.L. Albrecht et D.H. Hubbell. 1985. Rhizosphere bacteria and their use to increase plant productivity: a review. Agric. Ecosyst. Environ. 12: 99-116.

Grant, F.A., L.A. Glover, K. Killham et J.I. Prosser. 1991. Luminescence-based viable cell enumeration of Erwinia carotovora in the soil. Soil Biol. Biochem. 23: 1021-1024.

Grimes, H.D. et M.S. Mount. 1987. Influence of Pseudomonas putida on nodulation of Phaseolus vulgaris. Soil Biol. Biochem. 15: 127-132.

Höfte, M., M. Mergeay et W. Verstraete. 1990. Marking the rhizopseudomonas strain $7 \mathrm{NSK}_{2}$ with a $\mathrm{Mu} \mathrm{d}(\mathrm{lac})$ element for ecological studies. Appl. Environ. Microbiol. 56: 1046-1052.

Howell, C.R. et R.D. Stipanovic. 1979. Control of Rhizoctonia solani on cotton seedlings with Pseudomonas fluorescens and with an antibiotic produced by the bacterium. Phytopathology 69: 480-482.

Howell, C.R. et R.D. Stipanovic. 1979. Suppression of Pythium ultimum-induced damping-off of cotton seedlings by Pseudomonas fluorescens and its antibiotic, pyoluteorin. Phytopathology 69: 480-482. 
Howell, C.R., R.C. Beier et R.D. Stipanovic. 1988. Production of ammonia by Enterobacter cloacae and its possible role in the biological control of Pythium preemergence damping-off by the bacterium. Phytopathology 78: 1075-1078.

Howie, W.J. et T.V. Suslow. 1991. Role of antibiotic biosynthesis in the inhibition of Pythium ultimum in the cotton spermosphere and rhizosphere by Pseudomonas fluorescens. Mol. Plant-Microbe Interact. 4: 393-399.

Inbar, J. et I. Chet. 1991. Evidence that chitinase produced by Aeromonas caviae is involved in the biological control of soilborne plant pathogens by this bacterium. Soil Biol. Biochem. 23: 973-978.

Keel, C., U. Schnider, M. Maurhofer, C. Voisard, J. Laville, U. Burger, P. Wirthner, D. Haas et G. Défago. 1992. Suppression of root diseases by Pseudomonas fluorescens CHAO: importance of the bacterial secondary metabolite 2,4-diacetylphloroglucinol. Mol. Plant-Microbe Interact. 5: 413.

Kloepper, J.W. 1992. Plant growth-promoting rhizobacteria as biological control agents. Pages 255-274 in B. Metting (réd.), Soil microbial technologies. Marcel Dekker, New York.

Kloepper, J.W. et C. J. Beauchamp. 1992. Issues related to measuring colonization of plant roots by bacteria. Can. J. Microbiol. 38: 1219-1232.

Kloepper, J.W., R. Lifshitz et R. M. Zablotowicz. 1989. Free-living bacterial inocula for enhancing crop productivity. Trends Biotechnol. 7: 39-44.

Kloepper, J.W., R.M. Zablotowicz, E.M. Tipping et R. Lifshitz. 1991. Plant growth-promoting mediated by bacterial rhizosphere colonizers. Pages 315-326 in D.L. Keister et P.B. Cregan (réds.), The rhizosphere and plant growth. Kluwer Academic Publishers, Netherlands.

Kloepper, J.W., R. Rodríguez-Kábana, J.A. Mclnroy et R.W. Young. 1992. Rhizosphere bacteria antagonistic to soybean cyst (Heterodera glycines) and root-knot (Meloidogyne incognita) nematodes: Identification by fatty acid analysis and frequency of biological control activity. Plant Soil 139: 75-84.

Kluepfel, D.A., E.L. Kline, T.A. Hughes, H.D. Skipper et D.T. Gooden. 1990. Field testing of a genetically engineered rhizosphere inhabiting pseudomonad: development of a model system. Pages 189-199 in D.R. MacKenzie et S.C. Henry (réds.), Biological monitoring of genetically engineered plants and microbes. Agricultural Research Institute, Bethesda, MD.
Kùc, J. 1987. Plant immunization and its applicability for disease control. Pages 255274 in I. Chet (réd.), Innovative approaches to plant disease control. John Wiley and Sons, New York.

Legocki, R.P., M. Legocki, T.O. Baldurin et A.A. Szalay. 1986. Bioluminescence in soybean root nodules: demonstration of a general approach to assay gene expression in vivo by using bacterial luciferase. Proc. Nat. Acad. Sci. USA 83: 9080-9084.

Leong, J. 1986. Siderophores: their biochemistry and possible role in the biocontrol of plant pathogens. Annu. Rev. Phytopathol. 24: 187-209.

Lifshitz, R., J.W. Kloepper, M. Kozlowski, C. Simonson, J. Carlson, E.M. Tipping et I. Zaleska. 1987. Growth promotion of canola (rapeseed) seedlings by a strain of Pseudomonas putida under gnotobiotic conditions. Can. J. Microbiol. 33: 390-395.

Lindström, K., P. Lipsanen et S. Kaijalainen. 1990. Stability of markers used for identification of two Rhizobium galegae inoculant strains after five years in the field. Appl. Environ. Microbiol. 56: 444-450.

Loper, J. E. et J. S. Buyer. 1991. Siderophores in microbial interactions on plant surfaces. Mol. Plant-Microbe Interact. 4: 5-13.

Mahaffee, W.F., P.A. Backman et J.J. Shaw. 1991. Visualization of root colonization by rhizobacteria using a luciferase marker. Pages 248-251 in C. Keel, B. Koller et G. Défago (réds.), Plant growth-promoting rhizobacteria-progress and prospects. IOBCMPRS Bull. XIV/8.

Meighen, E. A. 1991. Molecular biology of bacterial bioluminescence. Microbiol. Rev. 55: 123-142.

O'Kane, D.J., W.L. Lingle, J.E. Wampler, M. Legocki, R.P. Legocki et A.A. Szalay. 1988. Visualization of bioluminescence as a marker of gene expression in Rhizobiuminfected soybean root nodules. Plant Molec. Biol. 10: 387-399.

Polonenko, D. R., F. M. Scher, J. W. Kloepper, C. A. Singleton, E. M. Laliberté et I. Zaleska. 1987. Effects of root colonizing bacteria on nodulation of soybean roots by Bradyrhizobium japonicum. Can. J. Microbiol. 33: $498-503$.

Schippers, B., A.W. Bakker, P.A.H.M. Bakker et R. van Peer. 1991. Beneficial and deleterious effects of $\mathrm{HCN}$-producing pseudomonads on rhizosphere interactions. Pages 211-219 in D.L. Keister et P.B. Cregan (réds.), The rhizosphere and plant growth. Kluwer Academic Publishers, Netherlands. 
Seong, K.-Y., M. Höfte, J. Boelens et W. Verstraete. 1991. Growth, survival, and root colonization of plant growth beneficial Pseudomonas fluorescens ANP15 and Pseudomonas aeruginosa 7NSK2 at different temperatures. Soil Biol. Biochem. 23: 423-428.

Shaw, J.J., P. Rogowsky, T.J. Close et C.I. Kado. 1987. Working with bacterial bioluminescence. Plant Mol. Biol. Rep. 5: 225236.

Shaw, J.J., F. Dane, D. Geiger et J.W. Kloepper. 1992. Use of bioluminescence for detection of genetically-engineered microbes released into the environment. Appl. Environ. Microbiol. 58: 267-273.

Sneh, B. 1981. Use of rhizosphere chitinolytic bacteria for biological control of Fusarium oxysporum f. sp. dianthi in carnation. Phytopathol. Z. 100: 251-256.

Suslow, T.V. 1982. Role of root-colonizing bacteria in plant growth. Pages 187-222 in M.S. Mount et G.H. Lacy. (réds.), Phytopathogenic prokaryotes. Vol. 1. Academic Press, New York.

Thomashow, L.S. et D.M. Weller. 1990. Application of fluorescent pseudomonads to control root diseases of wheat and some mechanisms of disease suppression. Pages 109-122 in D. Hornby (réd.), Biological control of soil-borne plant pathogens. CAB International, Wallingford, Oxon, U.K. van Peer, R., H.L.M. Punte, L.A. de Weger et B. Schippers. 1990. Characterization of root surface and endorhizosphere pseudomonads in relation to their colonization of roots. Appl. Environ. Microbiol. 56: 24622470.

van Peer, R., G.J. Niemann et B. Schippers. 1991. Induced resistance and phytoalexin accumulation in biological control of Fusarium wilt of carnation by Pseudomonas sp. strain WCS417r. Phytopathology 81: 728-734.

Wei, G., J.W. Kloepper et S. Tuzum. 1991. Induction of systemic resistance of cucumber to Colletotrichum orbiculare by selected strains of plant-growth-promoting rhizobacteria. Phytopathology 81: 15081512.

Weller, D. M. 1988. Biological control of soilborne plant pathogens in the rhizosphere with bacteria. Annu. Rev. Phytopathol. 26: 379-407. 